\title{
Highly optimized transitions to turbulence ${ }^{1}$
}

\author{
$\begin{array}{lll}\text { K. M. Bobba } & \text { B. Bamieh } & \text { J.C. Doyle }\end{array}$
}

\begin{abstract}
We study the Navier-Stokes equations in three dimensional plane Couette flow geometry subject to streamwise constant initial conditions and perturbations. The resulting two dimensional/three component $(2 \mathrm{D} / 3 \mathrm{C})$ model has no bifurcations and is globally (non-linearly) stable for all Reynolds numbers $R$, yet has a total transient energy amplification that scales like $R^{3}$. These transients also have the particular dynamic flow structures known to play a central role in wall bounded shear flow transition and turbulence. This suggests a Highly Optimized Tolerance (HOT) model of shear flow turbulence, where streamlining eliminates generic bifurcation cascade transitions that occur in bluff body flows, resulting in a flow which is stable to arbitrary changes in Reynolds number but highly fragile in amplifying arbitrarily small perturbations. This result indicates that transition and turbulence in special streamlined geometries is not a problem of linear or nonlinear instability, but rather a problem of robustness.
\end{abstract}

\section{Introduction}

Hydrodynamic stability theory (in both its linear and non-linear forms) provides excellent predictions of transition Reynolds numbers and scenarios for a variety of well studied flows such as Rayleigh-Benard convection and Taylor-Couette flow [11]. Statistical theories and various turbulence models provide good predictions of the statistics of fully-developed, homogeneous, isotropic turbulence $[1,2]$. More elusive is the case of wall bounded, high shear flows in which both transition and turbulence modelling appears to be significantly more difficult. The nearly stream-wise constant, elongated structures that play a dominant role in transition and turbulence in wall bounded high shear flows $[18,21]$ are not well explained by the standard stability transition or isotropic turbulence theories. Recent research $[23,20,3]$ has shown that a non-modal analysis

\footnotetext{
${ }^{1}$ This work is funded under AFOSR MURI grants: "Mathematical Infrastructure for Robust Virtual Engineering" and "Unified Theory for Complex Biological and Engineering Networks."

${ }^{2}$ Graduate Aeronautical Laboratories, California Institute of Technology, Pasadena, CA 91125

${ }^{3}$ Mechanical Engineering, University of California, Santa Barbara, CA 93106

${ }^{4}$ Control and Dynamical Systems, California Institute of Technology, Pasadena, CA 91125
}

of the linearized Navier-Stokes equations yields a much better description of transition, but attempts at finding a so-called "self-sustaining mechanism" $[26,27,28]$ are still inconclusive. We will explore attempts to reconcile these apparent contradictions between theory and experiment in perhaps the simplest and most extreme case, that of plane Couette flow.

\section{Main results}

The standard incompressible Navier-Stokes equations and boundary conditions in this case are

$$
\begin{aligned}
V_{t}+(V . \nabla) V & =-\nabla p+(1 / R) \Delta V \\
\nabla . V & =0
\end{aligned}
$$

with flow fields $V=(u, v, w)$, pressure $p$, and (1) is the momentum equations, $R$ is the Reynolds number, and (2) is the continuity equation. The no slip boundary conditions for normalized plane Couette flow are

$$
u(x, \pm 1, z, t)= \pm 1,0=v(x, \pm 1, z, t)=w(x, \pm 1, z, t)
$$

which mean that the channel walls are at $y= \pm 1$, while the streamwise $(x)$, and spanwise $(z)$ directions are infinite, i.e. $-\infty<x<\infty,-\infty<z<\infty$. The steady laminar flow is $u(x, y, z, t)=y$, and $v=w=0$.

Recent studies of linearized Navier-Stokes (LNS) equations for plane Couette (CLNS) flow indicate a consistent picture of transition as a process where stable, but noise amplifying flows robustly produces streamwise vortices and streaks. As in $[10,7]$, it can be shown that while CLNS flows are stable for all Reynolds numbers $R$, the total perturbation energy grows like $R^{3}$. This explosive amplification also generically produces nearly streamwise constant vortices and streaks of the type that are found ubiquitously in high shear flow experiments. This is consistent with the well-known experimental difficulties of building sufficiently quiet apparatus to produce high $R$ laminar plane Couette flow, and the observations of streamwise structures in high $R$ flows in pipes and boundary layers. Other work supports this view. Couette flow does not appear to have any known linear or non-linear instabilities $[4,15,16]$ nor does it have known attractors apart from the laminar flow $[5,6,14]$. The well known work on non-normal transient growth $[20,23,24,19,25]$ of the LNS in channel flow has shown that the modes with largest energy 
growth are streamwise constant. Pseudo-spectral analysis [22] of LNS shows the high sensitivity of these equations to dynamical perturbations, again with worstcase being streamwise constant perturbations.

Difficult questions remain however regarding the validity of this linearized picture for nonlinear regimes, especially since $R^{3}$ energy growth means that high $R$ flow transients can be very large even for vanishingly small initial perturbations. This paper is a first step towards developing the required global, nonlinear view. Given that the linearized $R^{3}$ energy growth is not only largest for streamwise constant flows, but also drops off sharply with increasing streamwise variation [9], a natural starting point is to consider streamwise constant initial conditions in (1)-(2), which produce streamwise constant solutions. To derive this model, we take the above NS equations and set all partial derivatives with respect to the stream-wise direction ( $x$ in our geometry) to zero. We call this the Two Dimensional/Three Component $(2 \mathrm{D} / 3 \mathrm{C})$ model since it represents the dynamics of the 3 component flow fields $u, v, w$ and pressure $p$ as functions of the two spanwise spatial variables $(y, z)$. It is convenient to recast these equations into the so-called evolution form by defining a "cross-sectional" stream function $\psi$ and rewriting (1) as

$$
\begin{aligned}
v & :=\psi_{z}:=\frac{\partial \psi}{\partial z}, \quad w:=-\psi_{y}:=-\frac{\partial \psi}{\partial y} \\
u_{t} & =-\psi_{z} u_{y}+\psi_{y} u_{z}+\frac{1}{R} \Delta u \\
(\Delta \psi)_{t} & =-\psi_{z}(\Delta \psi)_{y}+\psi_{y}(\Delta \psi)_{z}+\frac{1}{R} \Delta^{2} \psi \\
u( \pm 1, z, t) & = \pm 1, \psi_{z}( \pm 1, z, t)=\psi_{y}( \pm 1, z, t)=0(6)
\end{aligned}
$$

Now (2) is automatically satisfied if $v$ and $w$ are computed from $\psi$ using (3). Equations (4-5), together with the boundary conditions $(6)$ is our $2 \mathrm{D} / 3 \mathrm{C}$ model. We note that the second equation (5), which is independent of $u$, is exactly the equation for the stream function of a 2D fluid. The additional feature here (over a 2D fluid model) is equation (4) for the evolution of the third velocity component $u$. The stream function $\psi$ appears as a coefficient in this PDE, thus the dynamics of $\psi$ are coupled into the dynamics of $u$ but not visa versa.

A re-scaling of the equations gives a canonical form independent of $R$. Multiplying (4) by $R$ and (5) by $R^{2}$, and scaling time with $R^{-1}$ and $\psi$ with $R$ yields

$$
\begin{aligned}
U_{\tau} & =-\Psi_{z} U_{y}+\Psi_{y} U_{z}+\Delta U, \\
(\Delta \Psi)_{\tau} & =-\Psi_{z}(\Delta \Psi)_{y}+\Psi_{y}(\Delta \Psi)_{z}+\Delta^{2} \Psi,
\end{aligned}
$$

where $\tau:=t / R$ and

$$
U(\tau):=u(\tau R), \quad \Psi(\tau):=R \psi(s \tau R),
$$

with zero boundary conditions as in $(6)$, and no scaling in the spatial dimensions $y, z$. Note that these equations are independent of $R$. We will use this to ultimately show that while the non-linear $2 \mathrm{D} / 3 \mathrm{C}$ model is globally stable for all values of the parameter $R$, total transient energy growth scales like $R^{3}$. We begin by defining the kinetic energy of the (rescaled) fields $(v, w)$ in terms of the stream function $\Psi$

$$
\begin{aligned}
E_{\Psi}(\tau) & :=\frac{1}{2} \int_{-\infty}^{\infty} \int_{-1}^{1}\left[v^{2}+w^{2}\right] d y d z \\
= & : \frac{1}{2}\left\|v^{2}+w^{2}\right\|_{y, z}=\frac{1}{2}\left\|\Psi_{z}^{2}+\Psi_{y}^{2}\right\|_{y, z}
\end{aligned}
$$

where $\|\bullet\|_{y, z}$ is shorthand for the indicated integral. After some algebra it can be shown that this quadratic form is indeed a Lyapunov function for the system (8), i.e.

$$
\begin{aligned}
\dot{E}_{\Psi}(\tau) & =-\left\|\left(\Psi_{z z}\right)^{2}+2\left(\Psi_{z y}\right)^{2}+\left(\Psi_{y y}\right)^{2}\right\|_{y, z} \\
& <0
\end{aligned}
$$

and hence (8) is globally asymptotically stable. To show asymptotic stability of $(7)$, write $U=: \bar{U}+\tilde{U}$, where $\bar{U}=y$ is the plane Couette flow solution, so that equation (8) becomes

$$
\begin{aligned}
\tilde{U}_{\tau} & =-\Psi_{z} \tilde{U}_{y}+\Psi_{y} \bar{U}_{z}+\Delta \tilde{U}-\tilde{U}_{y} \Psi_{z} \\
0 & =\tilde{U}(y= \pm 1, z, \tau) .
\end{aligned}
$$

If we define the kinetic energy of $\tilde{U}$ by

$$
E_{\tilde{U}}(\tau):=\frac{1}{2}\left\|\tilde{U}^{2}\right\|_{y, z}:=\frac{1}{2} \int_{-\infty}^{\infty} \int_{-1}^{1} \tilde{U}^{2} d y d z,
$$

then after some algebra

$$
\dot{E}_{\tilde{U}}(\tau)=-\left\|\tilde{U}_{z z}^{2}+\bar{U}_{y y}^{2}+\Psi_{z} \tilde{U}\right\|_{y, z} .
$$

Stability becomes clear after noticing that $\dot{E}_{\bar{U}}$ is the same as if $\tilde{U}$ were governed by the equation

$$
\tilde{U}_{\tau}=\Delta \tilde{U}-\bar{U}_{y} \Psi_{z}
$$

which is equation (12) without the first two terms. In this last equation, $\Psi_{z}$ acts as an input to an asymptotically stable system, because the system $\tilde{U}_{\tau}=\Delta \tilde{U}$ is the heat equation with Dirichlet boundary conditions, and is therefore exponentially stable. Furthermore, the input $\Psi_{z}$ has exponentially decaying energy. These two facts imply that $\tilde{U}$ in equation (16) decays asymptotically to zero. This in turn implies that $\tilde{U}$ in equation (12) decays asymptotically to zero.

The previous analysis implies that both $E_{\Psi}$ and $E_{\tilde{U}}$ decay asymptotically to zero. $E_{\Psi}$ decays monotonically to zero, but $E_{\hat{U}}$ may increase in a transient manner before it asymptotically decays to zero. The final conclusion is that the kinetic energy $E_{\Psi}+E_{\tilde{U}}$ of the deviation from plane Couette flow decays asymptotically to zero from any initial condition of (7-8). Note that $E_{\Psi}+E_{\tilde{U}}$ is not a Lyapunov function for this system since it does not decay monotonically.

To study transient response to initial conditions, consider the Total Transient Energy Growth $\mathcal{E}_{T}$ defined by

$$
\mathcal{E}_{T}=\int_{0}^{\infty} E_{\psi}(t)+E_{\bar{u}}(t) d t .
$$


where we define $\tilde{u}(y, z, t)=u(y, z, t)-\bar{U}$ (the perturbation dependent on $R$ ) and $E_{\psi}(t)$ and $E_{\bar{u}}(t)$ in an analogous manner to (10) and (14). Clearly, $\mathcal{E}_{T}$ is a functional on the initial states of the system (4-5), i.e. $\mathcal{E}_{T}(\psi(0), u(0))$, and it describes an integral measure of the perturbation trajectory excursion given an initial condition perturbation. The following are immediate consequences of (9)

$$
\begin{aligned}
& E_{\tilde{U}}(0)=E_{\bar{u}}(0), \quad \int_{0}^{\infty} E_{\tilde{u}}(t) d t=R \int_{0}^{\infty} E_{\tilde{U}}(\tau) d \tau \\
& E_{\psi}(0)=\frac{1}{R^{2}} E_{\Psi}(0), \quad \int_{0}^{\infty} E_{\psi}(t) d t=\frac{1}{R} \int_{0}^{\infty} E_{\Psi}(\tau) d \tau .
\end{aligned}
$$

Furthermore, since the energy relations between $\Psi$ and $\bar{U}$ are captured by (16), we have that

$$
\int_{0}^{\infty} E_{\bar{U}}(\tau) d \tau=k_{c} \int_{0}^{\infty} E_{\Psi}(\tau) d \tau
$$

where $k_{c}$ is the square of the gain of the linear mapping between $\Psi$ and $\bar{U}$ in (16). This gain is non-zero due to the presence of nominal shear $\bar{U}_{y} \neq 0$. Using the definition of $\mathcal{E}_{T}$ and the relations above, we compute

$$
\begin{aligned}
\mathcal{E}_{T} & =R \int_{0}^{\infty} E_{\tilde{U}}(\tau) d \tau+\frac{1}{R} \int_{0}^{\infty} E_{\Psi}(\tau) d \tau \\
& =\left(k_{c} R+\frac{1}{R}\right) \int_{0}^{\infty} E_{\Psi}(\tau) d \tau .
\end{aligned}
$$

To compare this with the initial state's energy; we assume that $\tilde{u}(0)=0$, and compute

$$
\begin{aligned}
\frac{\mathcal{E}_{T}}{E_{\psi}(0)} & =\frac{\mathcal{E}_{T}}{\left(E_{\Psi}(0) / R^{2}\right)}=R^{2} \frac{\mathcal{E}_{T}}{E_{\Psi}(0)} \\
& =\left(k_{c} R^{3}+R\right) \frac{\int_{0}^{\infty} E_{\Psi}(\tau) d \tau}{E_{\Psi}(0)}
\end{aligned}
$$

and note that the last fraction is independent of $R$ since it involves $\Psi(\tau)$, which represents the dynamics evaluated at $R=1$. In particular, this last computation shows that the ratio between $\mathcal{E}_{T}$ and initial state energy scales like $R^{3}$. This happens for initial states for which $\psi(0) \neq 0$, but $\bar{u}(0)=0$. Note that the $R^{3}$ term is due to the subsequent growth in the energy of the $\tilde{u}$ term, while the corresponding growth in the $\psi$ term scales like $R$. More importantly, $R^{3}$ growth occurs when $k_{c} \neq 0$, which is the gain of the system (16) that represents the coupling from normal and spanwise velocity perturbations to streamwise velocity perturbations. It occurs due to the presence of the background shear $\bar{U}_{y}$. Roughly speaking, the $R^{3}$ growth is a combination of time dilation by $R$, and magnitude growth of $\tilde{u}^{2} \propto R^{2}$.

We summarize our results in the following theorem, for which the above argument is the sketch of a proof.

\section{Theorem 1 Consider the 2D/3C model (4-5)}

1. Plane Couette flow $\bar{U}=y$ is globally asymptotically stable for all Reynolds numbers $R$.

\section{For initial conditions $(\tilde{u}(0)=0, \psi(0) \neq 0)$}

$$
\mathcal{E}_{T}=k\left(k_{c} R^{3}+R\right) E_{\psi}(0)
$$

for some $k$ which is independent of $R$.

That the $2 \mathrm{D} / 3 \mathrm{C}$ model is globally stable at all Reynolds numbers partially explains the difficulties that researchers have encountered in trying to discover bifurcation transition routes to turbulence in $3 \mathrm{D}$ plane Couette flow. Our second result that total perturbation energy growth scales like $R^{3}$ in the non-linear $2 \mathrm{D} / 3 \mathrm{C}$ model further suggests that bifurcation transitions may not be required. While we have thus obtained striking and encouraging confirmation in our nonlinear $2 \mathrm{D} / 3 \mathrm{C}$ model of the observations made using the linearized $3 \mathrm{D} / 3 \mathrm{C}$ CLNS model, much more work is needed to complete a global nonlinear $3 \mathrm{D} / 3 \mathrm{C}$ picture of transition and turbulence. An obvious next step is to understand how the large transient $2 \mathrm{D} / 3 \mathrm{C}$ solutions evolve when perturbed away from their streamwise constant solutions within the full 3D/3C NS equations.

\section{Remarks}

Perhaps most importantly, the above results motivate the argument that to understand transition and turbulence in plane Couette flow, it is necessary to include an explicit uncertainty analysis. While we have focused on initial condition perturbations, the $2 \mathrm{D} / 3 \mathrm{C}$ model will have extreme sensitivities to external excitation and unmodeled dynamics as well. These can be thought of as forces that are external to the exact NS equations, and could arise from such things as thermal fluctuations, free stream disturbances, wall roughness, wall vibrations, and non-newtonian effects. We reviewed briefly how this uncertainty analysis can be performed on linearized versions of this model, and how this analysis leads to stream-wise vortices and streaks as the robustly dominant flow structures at high Reynolds numbers. Simulation has so far been our only tool to confirm these observations in the nonlinear $2 \mathrm{D} / 3 \mathrm{C}$ model. Further quantification of the sensitivity of the non-linear $2 \mathrm{D} / 3 \mathrm{C}$ model to unmodeled dynamics and external noise requires more technical machinery from robust control theory. These problems, as well as new experimental studies of the details of transitional flows, is the subject of current work $[9,12,13]$.

The globally stable but extremely sensitive high $R$ flows studied here perfectly illustrate the "robust, yet fragile" characteristic of Highly Optimized Tolerance (HOT), which arises in general when deliberate robust design aims for a specific level of tolerance to uncertainty. In $[8,17]$, the role of design in producing high yield percolation lattices was studied. Random lattices have low yield which is maximized at criticality, 
whereas highly designed HOT lattices can have high yields, but are hypersensitive to design flaws and modeling assumptions. In flows, an important design is streamlining for low drag. Plane Couette flow is merely an extreme example of a very streamlined flow geometry, and many of the conclusions herein should apply to pipes, wings, and other streamlined scenarios. For example, the optimization in a pipe could be based on maximum mass flow rate for a given pressure drop. An airfoil shape is designed to trade off maximum lift versus minimum drag within a range of speeds. Both designs can be thought of as moving from a generic state of randomly twisted and bumpy pipes and bluff bodies to a more structured HOT state of smooth, straight pipes and airfoils.

This streamlining eliminates bifurcation transitions caused by instability to uncertainty in initial conditions, allowing highly sheared flows to remain laminar to high Reynolds number, just as critical phase transitions are eliminated in designed lattices. The resulting flows, however, become extremely sensitive to new perturbations which were previously irrelevant, again exactly as for percolation lattices. For flows, these newly acquired sensitivities are huge amplifications of very small perturbations like wall roughness, vibrations and other disturbances and unmodeled dynamics. These "robust, yet fragile" features are characteristic of HOT systems, which universally have high performance and high throughput, but potentially extreme sensitivities to design flaws and unmodeled or rare perturbations. This paper supports the results in $[8,17]$ that strongly suggest that such HOT tradeoffs are inevitable consequences of high performance robust design, and are the central drivers in the complexity of engineering and biological systems. However, this emphasis on necessity and robustness is new and largely unexplored, particularly in the area of fluids, and this work is merely the first initial step in a new approach to design and control of unsteady flows.

\section{References}

[1] W. D. McComb, The Physics of Fluid Turbulence, Oxford University Press, (1990)

[2] M. Hallback, Ed., Turbulence and transition modelling, Kluwer, (1996)

[3] S. Grossmann, Rev. Mod. Phys. 72, 603, (2000)

[4] V. A. Romanov, Funkcional Anal. $i$ Prolozen 7, $62,(1973)$

[5] D. Ruelle and F. Takens, Commun. Math. Phys. 20, 167, (1971)

[6] A. Schmiegel and B. Eckhart, Phys. Rev. Lett. 79, 5250, (1997)

[7] B. Bamieh and M. Dahleh, Energy amplification in channel flows with stochastic excitation, Phys. Fluids 13,3258, (2001)

[8] J. M. Carlson and J. C. Doyle, Phys. Rev. E 60, 1412, (1999)

[9] M. Jovanovic and B. Bamieh, Input-output Analysis of the Linearized Navier-Stokes Equations, in preparation.

[10] B. F. Farrell and P. J. Ioannou, Phys. Rev. Lett. 72, 1188, (1994)

[11] P. G. Drazin, and W. H. Reid, Hydrodynamic Stability, Cambridge University Press, (1981)

[12] K. M. Bobba and J. C. Doyle, Uncertainty Analysis of Transition to Turbulence, to be submitted to Phys. Fluids

[13] K. M. Bobba, J. C. Doyle, and M. Gharib, Proceedings of 14th Australasian Fluid Mechanics Conference, 9-14 Dec, (2001)

[14] H. L. Swinney and J. P. Gollub, Physica D 18, 448, (1986)

[15] M. Nagata, J. Fluid Mech. 217, 519, (1990)

[16] R. M. Clever and F. H. Busse, J. Fluid Mech. 234, 511, (1992)

[17] J. M. Carlson and J. C. Doyle, Phys. Rev. Lett. 84, 2529, 2000

[18] S. J. Kline, W. C. Reynolds, F. A. Schraub and P. W. Runstadler, J. Fluid Mech. 30, 741, (1967)

[19] W. Orr, Proc. Roy. Irish Acad. A 27, 9, (1907)

[20] S. C. Reddy and D. S. Henningson, J. Fluid Mech. 252, 209, (1993)

[21] R. S. Rogallo, P. Moin, Annual Rev. Fluid Mech. 16, 99, 1984.

[22] L. N. Trefethen, A. E. Trefethen, S. C. Reddy, and T. A. Driscoll, Science 261, 578, (1993)

[23] K. M. Butler and B. F. Farrell, Phys. Fluids 4, 1637, (1992)

[24] L. H. Gustavsson, J. Fluid Mech. 224, 241, (1991)

[25] D. J. Benney and L. H. Gustavsson, Stud. Appl. Math. 64, 185, (1981)

[26] B. F. Farrell, P. J. Ioannou, Theoret. Comput.Fluid Dynamics 11, 237 (1998)

[27] J. S. Baggett and L. N. Trefethen, Phys. Fluids 9, 1043, (1996)

[28] F. Waleffe, Physical Review Letters 81, 4140, (1998) 